\title{
Infants attend to second-order relational properties of faces
}

\author{
L. A. THOMPSON, V. MADRID, S. WESTBROOK, and V. JOHNSTON \\ New Mexico State University, Las Cruces, New Mexico
}

\begin{abstract}
Two experiments investigated whether 7-month-old infants attend to the spatial distance measurements relating internal features of the human face. A visual preference paradigm was used, in which two versions of the same female face (one either lengthened or shortened, and one nonmodified) were presented simultaneously. In Experiment 1, infants looked longer at the nonmodified faces, which were determined to match the average distance relationships found in a sample of faces drawn from the same population. Longer looking times for modified faces were found in Experiment 2, in which the nonmodified faces were unusually long and the modified faces conformed to average distance measurements. It is proposed that infants' attention to the spatial relations of internal face features is an optimal tool for lifelong face recognition.
\end{abstract}

The human infant's ability to discriminate and recognize faces is a feat that inspires much curiosity and controversy among researchers. Borne of this fascination is a body of empirical research that helps lay to rest William James's (1890) characterization of the infant's perceptual world as one big "blooming, buzzing confusion" (p. 488) in favor of a more perceptually discerning infant. Much to our delight, even newborn infants will visually track a face pattern farther than scrambled face patterns (Johnson, Dziurawiec, Ellis, \& Morton, 1991), they show looking time preferences for their mothers' faces over strangers' faces (Bushnell, Sai, \& Mullin, 1989; Field, Cohen, Garcia, \& Greenberg, 1984; Pascalis, De Schonen, Morton, Deruelle, \& Fabre-Grenet, 1995), and they produce more sucking responses to mothers' faces than to strangers' faces (Walton, N. J. A. Bower, \& T. G. R. Bower, 1992). Astonishingly, within 6 days of birth, infants also show longer looking times for attractive female faces (as judged by adults) than to unattractive faces (Slater et al., 1998). Thus, newborn infants are not simply discerning; they also have "good taste."

A wide range of adult-rated faces elicit preferential looking in infants. For example, within the visual preference paradigm, 2- to 3-month-old (Langlois et al., 1987) and 6month-old (Langlois et al., 1987; Samuels, Butterworth, Roberts, Graupner, \& Hole, 1994; Samuels \& Ewy, 1985) Caucasian infants looked longer at attractive Caucasian

This research was supported in part by a grant to New Mexico State University from the Ronald E. McNair program. The authors thank Jennie Brown, Eunice Duran, Amanda Lindsay, Al Moreno, Paul Vonnahme, and Deborah Olcott for their assistance in creating stimuli or running the experiments. The authors also thank Gillian Rhodes, Susan Carey, John Wixted, and David Ostby for their critical feedback on the manuscript. Many thanks go to the parents and infants who participated in this study. Correspondence should be addressed to L. A. Thompson, Psychology Department (3452), Box 30001, New Mexico State University, Las Cruces, NM 88003 (e-mail: thompson@ crl.nmsu.edu). women's faces than at unattractive Caucasian women's faces. The attractiveness effect held across gender for adult male faces (Langlois, Ritter, Roggman, \& Vaughn, 1991; Samuels \& Ewy, 1985), across ethnicity when primarily Caucasian infants viewed Black female faces (Langlois et al., 1991), and across age when infants viewed infant faces rated as attractive or unattractive by adults (Langlois et al., 1991).

The question arises, on what basis are infants making these facial discriminations? Some studies conducted on infants 1-month-old or less suggest that the neonate's attention to the face may not actually include the internal facial features. For example, Pascalis et al. (1995) found that 4-day-old infants did not maintain a preference for their mothers over strangers when the women were wearing scarves that occluded the information from the hairline and outer contours of their heads. Salapatek (1975) showed that 1-month-old infants scan the external contours of the face (the chin and hairline) whereas 2-month-old infants concentrated on the interior-specifically, the eyes and mouth region. However, research also shows that internal features of the face (eyes, nose, mouth) are processed by young infants. For example, newborn infants' attractiveness preferences suggest that they are attending to the internal region of the human face (Langlois et al., 1987; Slater et al., 1998), and 3-month-old infants are sensitive to various facial expressions in photographs of their mothers (Barrera \& Maurer, 1981). Thus, neonates may arrive "equipped" with a predisposition to process face-like patterns (Johnson et al., 1991), attending to the interior region of the face right from birth or shortly thereafter. However, although young infants are sophisticated face processors by any measure, there is still room for growth. In infants as old as 6 months, the visual preference for mother over a stranger has been found to depend on the difficulty of discrimination between face pairs (de Haan \& Nelson, 1997). 
How are faces processed by experts? There is growing evidence that adults recognize faces in a manner different from other classes of objects (Farah, Wilson, Drain, \& Tanaka, 1998), using some type of processing of the overall structure or configuration of the face (cf. Rhodes, 1988; Rhodes, Brennan, \& Carey, 1987; Valentine \& Bruce, 1986). The specific type of processing involved is a matter of controversy (for a review of theories of face perception, see Farah et al., 1998). One well-known view was offered by Diamond and Carey (1986). They distinguish between first- and second-order relational properties used in object recognition. Much of human pattern recognition can be construed as the encoding and representation of objects and parts of objects in spatial relationship to each other. For example, houses on the block may have driveways on the right side or the left side of the lot and varying numbers of trees, rocks, or cacti in different locations. First-order relational information is the arrangement of these parts without reference to a prototypical arrangement. Diamond and Carey argue that human faces are encoded in terms of first-order spatial relations between elements sharing a configuration (e.g., the left pupil, right pupil, nose, mouth, bottom of chin, etc.), but, since faces all have a common configuration of elements, they are individuated in terms of their second-order relational properties. Second-order relational properties are the "distinctive relations among the elements that define the shared configuration" (Diamond \& Carey, 1986, p. 110). Thus, in comparison with a face prototype, a particular face may have an unusually long eye-to-mouth distance.

The "face inversion effect" has been used to support the idea that humans acquire an expertise in face processing. The face inversion effect is the finding that face recognition is disrupted more by inversion during encoding than is recognition using other classes of canonically oriented stimuli (Yin, 1969). Diamond and Carey (1986) argue that the face inversion effect "will emerge whenever three conditions are met. First, the members of the class must share a configuration. Second, it must be possible to individuate the members of the class on the basis of secondorder relational features. Third, subjects must have the expertise to exploit such features" ( p. 116). They found that dog judging experts, but not nonexperts, showed an inversion effect for photographs of dogs comparable in magnitude to the face inversion effect, demonstrating that inversion effects are not limited to face stimuli and that expertise is required before pronounced inversion effects will be obtained.

Some evidence exists to support the idea that adults differentiate faces and other objects on the basis of secondorder relationships (e.g., Diamond \& Carey, 1986; Yin, 1969). Moreover, consistent with the often-cited 10-year period for the development of expertise (e.g., Ericsson \& Smith, 1991), the face inversion effect has been demonstrated to increase across childhood until around the age of 10 (e.g., Carey \& Diamond, 1977; Carey, Diamond, \& Woods, 1980). More recently, Carey and Diamond (1999) argue that face encoding on the basis of second-order relational features is a necessary, but not a sufficient, marker of expertise in face processing.

One question that arises is, do infants show a sensitivity to second-order relations? Our goal in the present study was to determine whether or not 7-month-old infants are sensitive to second-order relational features in face viewing. Determining that infants of this age have the ability to make second-order relational discriminations between facial elements would indicate that they possess a cognitive skill that Carey and Diamond (1999; Diamond \& Carey, 1986) argue is necessary to launch them on the road to eventually becoming experts in face processing.

A related line of research also proposes that adults and infants process faces in terms of their similarity to an averaged prototype of the face category. Furthermore, instances that are closest to the average prototype are deemed "most attractive" by adults (Langlois \& Roggman, 1990; Rhodes, Sumich, \& Byatt, 1999; Rhodes \& Tremewan, 1996) and are looked at longer by infants (Rubenstein, Kalakanis, \& Langlois, 1999), relative to individual faces. Rubenstein et al. claim that a cognitive averaging process is used by infants to form a face prototype and is also used by infants during the actual visual preference task when a new face is compared to the prototype. In their model, prototype formation is based on the "hundreds of dimensions" (Rubenstein et al., 1999, p. 851) that vary in naturalistic face stimuli. The procedure used in their research to create an averaged face involved mathematically averaging hundreds of facial dimensions within a 32-stimulus set of black-and-white photographs of young Caucasian women (e.g., Langlois \& Roggman, 1990; Langlois, Roggman, \& Musselman, 1994; Rubenstein et al., 1999). Secondorder relational properties are among this set, but so are many, many other properties, such as skin tone, hair color, hair length, hair contour, and the shapes of the eyes, mouth, chin, ears, and eyebrows, to name a few examples. Germane to the present study is the issue of whether or not the entire set of hundreds of possible facial properties represented within a 32-face composite is actually needed in the prototype comparison process. If it can be shown that infants are sensitive to the properties manipulated within the face stimuli of the present study, then we have evidence that only a subset of all theoretically possible face properties are required for face discrimination.

In the present study, we investigated 7-month-old infants' abilities to discriminate the nonindependent second-order relational properties of the human face, which are changed when the eye-to-mouth and eye-to-chin distances are either lengthened or shortened. Black-and-white photos were modified so that the region of the face between the pupil and the mouth was either lengthened or shortened by a constant distance. In a standard visual preference paradigm, modified and nonmodified versions of the same adult female face were presented simultaneously for infant viewing. Thus, in all respects, the faces being compared on any given trial were identical, except for the 
properties that were altered by changing the eye-to-mouth distances. These would include the length of the jawline itself, the length of the nose, and other distance relationships that connect the mouth and chin with other features.

Evidence supporting infants' ability to discriminate on the basis of this second-order relational information would be apparent if they showed longer looking times toward either the modified or the nonmodified faces. Support for the notion that infants prefer average eye-to-mouth and eye-to-chin distance parameters would be evident in longer looking times toward nonmodified faces relative to both classes of modified faces.

\section{EXPERIMENT 1}

\section{Method}

Participants. Fifty-four 6- to 8-month-old infants participated in this experiment. They were recruited through the introductory psychology subject pool at New Mexico State University and by posting fliers at daycare centers and business establishments throughout Las Cruces, NM. Fifteen infants were eliminated from the final sample for the following reasons: 5 were too fussy to complete the task, 2 refused to look at the projection screens, 1 had a left-side bias, 3 had a rightside bias (on at least half of the trials, they did not look at all toward the left side), one parent interfered with the baby's ability to see the screens, and 3 were eliminated for experimenter error. Of the 39 remaining infants in the final sample, there were 17 males and $22 \mathrm{fe}-$ males; 20 were Hispanic ( 8 males, 12 females), 16 were Caucasian ( 8 males, 8 females), and 3 were Hispanic-Caucasian ( 1 male, 2 females). Their ages ranged from 24 to 35 weeks ( $M=29.5$ weeks). All infants were full-term at birth and were healthy at the time of the experiment.

Stimuli. Black-and-white photographs were made of four Hispanic and five Caucasian undergraduate females (each roughly 21 years old). Each woman was photographed at a constant distance and lighting and wore no make-up or jewelry. Their hair was brushed back or pulled back so that their faces were in full view, but several had bangs on their foreheads. Photos were scanned into a Macintosh computer and transported into image-processing software (Adobe Photoshop 3.0). Each photo image was scaled to an interpupil distance of 55 pixels and was "touched up" by smoothing out skin blemishes or shadowing. These are referred to as the nonmodified stimuli. For eight of the nine images, a modified version was also created by either adding ( 4 faces) or splicing out (4 faces) several narrow horizontal sections of the interior region of the face, between the eye (middle of the pupil) and the mouth (top of the lips), by a total of $3 \mathrm{~mm}$. The 3-mm difference was a measurement taken when the photo was printed on paper in a $3 \frac{1}{4} \times 3 \mathrm{in}$. format. The ninth face was not modified because it served as a practice stimulus. A smooth, natural-looking jawline was brushed in using the software tool. All other features of the face were left intact. Black-and-white printouts were made from the computer files and were then made into slides. The size of the face as it appeared from the infant's position on the projection screen was approximately $25 \%$ larger than life size. Example photographs used in the experiment appear in Figure 1.

In order to assess whether the lengthened and shortened versions of the faces were perceived to be different from the norm in an adult population, we collected typicality ratings of the eye-to-chin distances. If the lengthened and shortened face stimuli are perceived to be different from a typical face on the length dimension, significant differences in typicality ratings should be obtained between the lengthened stimuli and their nonmodified face counterparts and between the shortened stimuli and their nonmodified face counterparts. Twenty-three undergraduate students from a course in cognitive psychology (13 females and 10 males) rated the 17 stimuli on a 7-point Likert scale "according to how typical the eye-to-chin distance is for a college-aged female," with 1 representing extremely atypical (short), 4 representing a typical length, and 7 representing extremely atypical (long). The average typicality ratings were $2.9(S D=0.6$; range $=1.00-4.00)$ for the shortened faces and $4.1(S D=0.5$; range $=3.25-4.75$ ) for the nonmodified versions, which was a significant difference $[t(22)=-7.61, p<.0001]$. The average typicality ratings were $5.7(S D=0.5$; range $=4.78-6.75)$ for the lengthened faces and $4.2(S D=0.5$; range $=3.25-5.00)$ for the nonmodified versions, which was also a significant difference $[t(22)=12.12, p<.0001]$.

Face parameter measurements. Several distance parameters of the modified and nonmodified faces were evaluated against a comparable set of 32 faces from the same population of young female undergraduates at New Mexico State University. Black-and-white photographs of the faces were scanned into the computer, and each was adjusted to an interpupillary distance of 55 pixels. By counting pixels, three measurements of each face were taken: (1) pupil to lower brow, (2) pupil to mouth, and (3) pupil to chin (for a visual description of the way the measurements were taken, see Johnston \& Franklin, 1993, p. 190). In each case, a vertical line was drawn from the midpoint of the left pupil to the horizontal line drawn from the midpoint of each location. All face measurements were defined by the distance (in pixels) between the pupil and the intersecting point.

Table 1 presents this information. The reference group of faces represents the comparison group of 32 faces. The three distance parameters taken from this set did not differ significantly from the nonmodified faces used in the present study, indicating that our nonmodif ied set included distance parameters representing the mean of the population of young women from the surrounding community. The eye-to-brow measurements did not differ significantly between the reference group and the shortened and the lengthened group, which is as it should be, since the modifications were made below the eye region. In the shortened face group, both the eye-tomouth and eye-to-chin distance parameters were significantly shorter than for the reference group. In the lengthened face group, the average eye-to-mouth distance was significantly longer than for the reference group. The average eye-to-chin distance in the lengthened face group was longer, but not significantly so, relative to the reference group.

Table 1

Mean Numbers (and Standard Deviations) of Pixels Between the Middle of the Pupil and Other Face Points and Corresponding $t$ Values for the Difference Between the Reference Group Faces and the Other Three Groups of Faces

\begin{tabular}{|c|c|c|c|c|c|c|c|c|c|c|}
\hline \multirow[b]{2}{*}{ Face Group } & \multirow[b]{2}{*}{$n$} & \multicolumn{2}{|c|}{ Brow } & \multirow[b]{2}{*}{$t$} & \multicolumn{3}{|c|}{ Mouth } & \multicolumn{3}{|c|}{ Chin } \\
\hline & & $M$ & $S D$ & & $M$ & $S D$ & $t$ & $M$ & $S D$ & $t$ \\
\hline Reference & 32 & 9.5 & 2.3 & & 60.2 & 5.1 & & 100.2 & 8.1 & \\
\hline Nonmodified & 9 & 11.2 & 3.2 & 1.76 & 57.6 & 3.8 & -1.46 & 97.1 & 6.5 & -1.05 \\
\hline Shortened & 4 & 11.0 & 4.0 & 1.09 & 52.0 & 0.8 & $-3.19 * *$ & 90.5 & 1.7 & $-2.36 * *$ \\
\hline Lengthened & 4 & 11.3 & 3.1 & 1.34 & 66.3 & 5.0 & $2.24 *$ & 106.0 & 9.6 & 1.32 \\
\hline
\end{tabular}

$* p<.05 . * * p<.01$. 

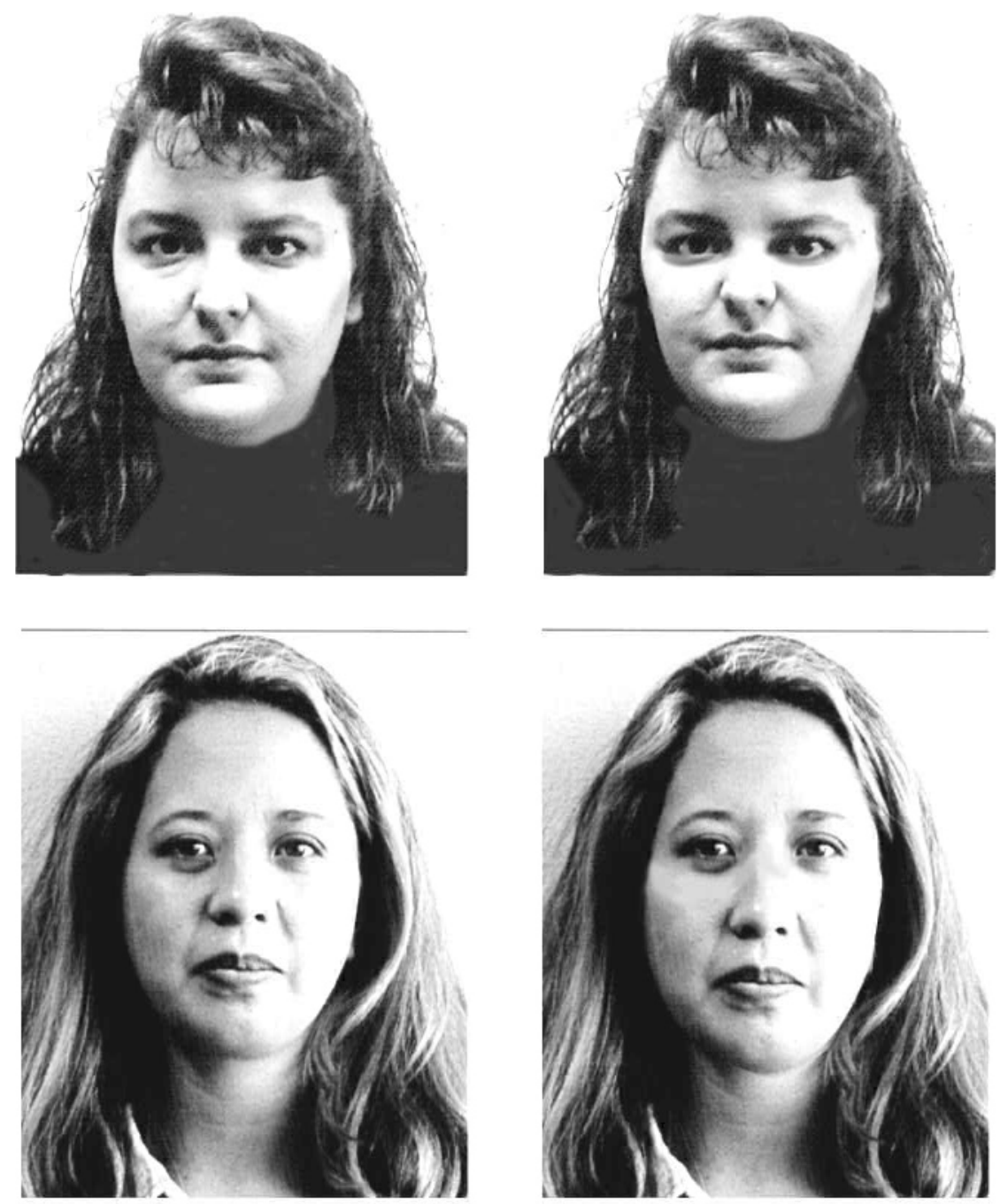

Figure 1. Photographs of females used as stimuli in the experiment. The two photos on the left are nonmodified. The face on the upper right was shortened, and the face on the lower right was lengthened.

Design. The first 2 trials in each 18-trial set were practice trials, in which the same nonmodified face appeared at both the right projection screen and the left projection screen. There were five different randomized orderings of the remaining 16 trials, with approximately 10 infants viewing each set. Within each set, a modified version and a nonmodified version of the same face were presented simultaneously. There were four pairs containing a lengthened modified face and four pairs containing a shortened modified face, and each face pair was shown two times. The modified version of each pair was presented once on the infant's right side and once on the left side. The only constraint in ordering the sets was that no fewer than 2 trials occurred between presentations of the same face pair.

Apparatus and Procedure. A standard visual preference paradigm was used. Slide pairs were rear-projected by Kodak carousel projectors (Model $650 \mathrm{H}$ ) onto two screens positioned at slight angles in front of the infant. The right side of the left image and the left side of the right image were $70 \mathrm{~cm}$ apart. The infant sat on his/her parent's lap $100 \mathrm{~cm}$ away from the inner angles of the screens. At this distance, the image subtended an average $14.6^{\circ}$ of visual angle. A lamp was situated in the midline position overhead and near the screens so that the infant could remain adapted to the brightness of the slides from within the darkened room. A small red light also appeared at midline at the infant's eye level. This light flashed on and off between trials and, along with a ringing cowbell, was used to orient the infant toward center prior to the beginning of each trial. The infant controlled the beginning of each trial by orienting toward the center for $2 \mathrm{sec}$.

The 10-sec trial length, slide advancement, and data recording were controlled by a PC-based computer with a 286 processor. One experimenter stood behind a barrier at the center and looked through a one-way mirror at the infant, recording the amount of time the infant looked toward the right, center, or neither position. The recording was done by hand-held switches. The experimenter who operated the switches could not see the screens that the infants were viewing and was unaware of the slide order. A second experimenter ordered the slides and initiated the experimental trials. This person viewed the infant on a monitor that was fed by a signal from a video camera mounted behind the barrier in front of the infant. During the exper- 
iment, the parents wore lightweight goggles, which were painted black, so that they could not see the stimuli and potentially influence the looking preferences of their infants.

As in Langlois et al. (1987), we obtained interobserver reliability of visual-fixation scoring by having each experimenter view 13 prerecorded infant sessions from a pilot experiment using exactly the same methodology as the present experiment, scoring the infants' looking times to their right and left sides across 18 trials. The average of the reliabilities across the four experimenters conducting this study was .93, using Cronbach's alpha.

\section{Results}

Trials for which the infant looked at neither the right side nor the left side were discarded (5\% of trials) prior to computing the average looking time for each infant and each face. Each infant's looking times were averaged across the two presentations of each stimulus (presented at the infant's right and left sides), excluding practice trials. Four sets of average looking times were created: two for the nonmodified face complements of the shortened and lengthened face pairings, and one each for the shortened and lengthened faces. Looking times were analyzed with a $2 \times 2$ analysis of variance, with two levels of modification type (modified vs. nonmodified) and two levels of alteration (shortened vs. lengthened). Both variables were within-subjects factors. The average looking times for modified and nonmodified faces from both types of face pairs can be seen in Figure 2.

The results showed that the infants looked significantly longer at the nonmodified faces than at the modified faces $[F(1,38)=5.05, p<.05]$. The main effect of type of alteration was not significant $(F=2.33, p>.05)$, nor did it interact with modification type $(F=1.13, p>.05)$.

\section{EXPERIMENT 2}

An alternative explanation for the effect exhibited by infants in Experiment 1 is that they can discriminate between faces that have been digitally altered and faces that have not been altered, and they prefer the unaltered faces. Alterations to the faces used as stimuli in Experiment 1 were not apparent to adult viewers during pilot testing. However, it is possible that the infants were able to pick up on minute variations made to the original faces by the digital alteration procedure and used this as the basis for their discrimination, instead of the second-order relational information involved in altering eye-to-chin and eye-tomouth distances. The primary purpose of Experiment 2 was to determine whether infants prefer modified faces over nonmodified faces when the nonmodified faces lie on the extreme end of the vertical elongation dimension and the modified faces are structurally closer to average spatial relations than are the nonmodified faces. This evidence would help to strengthen the argument that the basis for infants' preferences is the encoding of distance relations between critical features of the face.

A second purpose of Experiment 2 was to determine whether infants' preference for average spatial relations would generalize to a different set of faces containing only modified faces that have been shortened, rather than those that have been both lengthened and shortened, as was the case in Experiment 1. Although the effect of type of alteration (lengthening vs. shortening) was not significant in Experiment 1 , the absolute difference in looking time between modified and nonmodified faces was greater for lengthened faces than for shortened faces. Thus, it would be use-

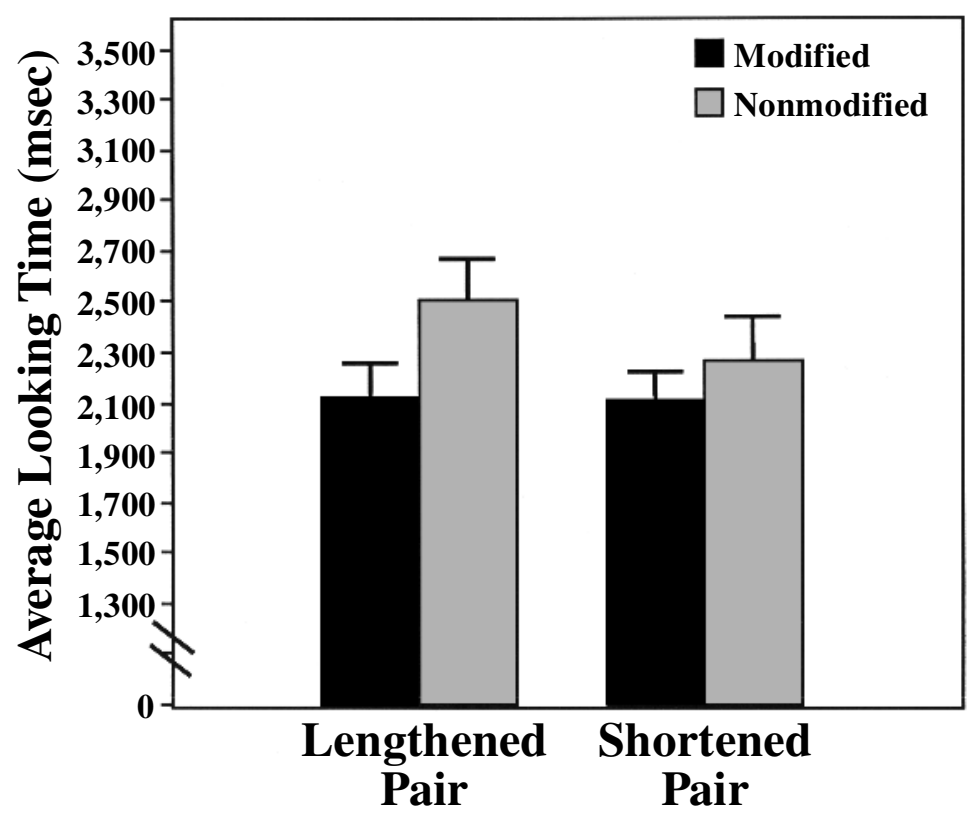

Figure 2. Average looking times to modified and nonmodified faces for both types of stimulus pairs in Experiment 1. Bars indicate 1 standard error. 
ful to determine whether the preference for average faces can be clearly shown in a set of faces that have been only shortened.

To this end, we used the same visual preference methodology with a second group of 6- and 7-month-old infants. The altered faces were modified only in the direction of shortening the distance between eye-to-mouth and eye-tochin regions. Two face pairs included nonmodified faces that were 1 standard deviation longer than the norm; the remaining pairs included faces whose nonmodified spatial relation parameters were closer to the average parameters than were the modified faces in the set.

\section{Method}

Participants. Twenty-two 6- and 7-month-old infants participated in Experiment 2. Three infants were eliminated from the final sample due to fussiness, and 1 infant was eliminated due to a left-side looking bias. The final sample of 18 infants included 9 males and 9 females, ranging from 21 and 28 weeks ( $M=25.6$ weeks). As in Experiment 1 , they were healthy, full-term infants of Hispanic ( 3 males and 4 females), Caucasian (4 males and 4 females), and HispanicCaucasian ( 2 males and 1 female) ethnic origin. Since there were no 8-month-old infants in this sample, the average age of the infants tested was 4 weeks younger than that of the infants in Experiment 1.

Stimuli. The sample of young females photographed for the experiment included four Caucasians, two Hispanics, one Amerasian, and two Caucasian-Hispanics from New Mexico State University. Setup of the models and method of scanning black-and-white photos into the computer was the same as for Experiment 1. Each photo image was scaled to an interpupil distance of 55 pixels, and modifications to eight stimuli were made to create shortened versions of the faces. The ninth face was not modified and was included as a practice stimulus.

The reference group of 32 faces of women from the surrounding community contained an average eye-to-mouth distance (pixels) of $60.2(S D=5.1)$ and an average eye-to-chin distance of $100.2(S D=8.1)$. Two of the nonmodified faces contained measurements for these parameters that were at least 1 standard deviation longer than the norm on one parameter (in the case of one face) or on both parameters (in the case of the other face). The modified versions of these faces fell within the range of the average distance measurements (57.0 and 95.5 pixels for eye-to-mouth and eye-to-chin measurements, respectively).

The average eye-to-mouth distance (pixels) for the remaining nonmodified faces was $57.3(S D=1.5)$ and the average eye-to-chin distance was $94.9(S D=1.6)$. The shortened versions of these faces contained an average eye-to-mouth distance of $55.7(S D=1.4)$ and an average eye-to-chin distance of $91.8(S D=1.3)$. Both shortened and nonmodified sets of faces used in this experiment included a narrower range of distance relations than did the reference group.

Design. As in Experiment 1, the infants viewed 18 sets of faces. The first 2 trials were practice trials, in which the same face was presented on right and left sides. There were five randomized orderings of the 16 experimental trials, with approximately the same number of infants receiving each order. Within each block of trials, the order of appearance of the two naturally long nonmodified faces was determined randomly. Each face pair was shown twice, with the modified version of the original face appearing once on the infant's right side and once on the left side.

Apparatus and Procedure. The same equipment and procedure were used as in Experiment 1. As in Experiment 1, reliability was computed across 13 infants' data from a pilot study, and the average reliability for the two experimenters was .92, using Cronbach's alpha.

\section{Results}

Trials for which the infant looked at neither of the faces were eliminated before computing average looking times
( $1 \%$ of trials). Mean looking times were calculated across left and right presentations of the naturally long nonmodified faces ( 4 trials for each infant) and likewise for the modified versions of these face sets (4 trials). The mean looking times were $2,873 \mathrm{msec}(S D=958)$ for the naturally long nonmodified faces and $3,857 \mathrm{msec}(S D=1,378)$ for the shortened (but average) modified versions of these face pairs. Infants looked significantly longer at the modified faces in this set $[t(17)=2.73, p<.01]$.

The mean looking times across the remaining six pairs of faces were calculated in a likewise manner. The average of the infants' looking times were $3,454 \mathrm{msec}(S D=1,071)$ for nonmodified faces (12 trials for each infant) and $2,852 \mathrm{msec}(S D=757)$ for shortened versions of these faces, which was a significant difference $[t(17)=2.38, p<.05]$.

\section{DISCUSSION}

In this study, we investigated whether 7-month-old infants could discriminate photographs of two faces, one of which was modified so that the eye-to-mouth and eye-tochin distances were either slightly shorter or slightly longer than the typical distances on a young adult female face. In two experiments, infants looked longer at the nonmodified faces when these faces conformed to average distance measurements. Experiment 2 showed that this preference was not based on infants' picking up on cues resulting from the digital alteration process, since the infants also preferred modified faces from face pairings in which the nonmodified faces were unusually long. Their longer looking times to the faces with average distance relationships, regardless of the alternative face pairing presented, clearly demonstrate that 7-month-old infants have the ability to make fairly fine discriminations of faces. Their looking time preferences also imply the use of a computational mechanism that mirrors a distance averaging algorithm, because the preferred faces shared distance measurements between individual face features that were similar to the average distance measurements from a population of likeaged female faces within the community.

Researchers have claimed that infants form face prototypes in a cognitive averaging process (Rubenstein et al., 1999; Walton \& T. G. R. Bower, 1993). The present results are consistent with this general claim: however, they diverge in two important respects. The issues at hand concern the nature of the description of the prototypical face representation invoked by infants during face processing. To explain these issues, we must more carefully compare the digital averaging procedures used in the present experiments and in Rubenstein et al. (1999).

Rubenstein et al. (1999) used the methodology of Langlois and Roggman (1990) to create their mathematically average face stimuli. As outlined in Langlois and Roggman (1990, pp. 116-117), this methodology began with a large set $(n=214)$ of black-and-white photos of mostly Caucasian female faces (ages were not specified), from which 96 faces were randomly selected and divided into three sets of 32. A standardized photographing procedure controlled factors such as lighting, facial expression, clothing style, 
and so on. Each photograph was scanned into the computer, and the faces were all scaled "by matching the location of the eye pupils and the middle of the lip line across all faces. A $512 \times 512$ matrix of numeric gray values then represented each facial image. By arithmetically averaging those matrices, a series of achromatic composite facial images was created" (Langlois \& Roggman, 1990, p. 116), one of which was a 32-face composite. Double edges sometimes appeared, and these were smoothed by adjusting each pixel value in the matrix to the average value of the surrounding pixels. Contrast was enhanced by adjusting the resulting range of gray values.

The first issue concerns our claim that second-order relational features involved in eye-to-mouth distance measurements are invoked in the prototype matching process. Our results suggest that 7-month-old infants can recruit their computing capabilities to operate on these properties of the face. Is this the same computational process that must underlie infants' abilities to discriminate faces in Rubenstein et al.'s (1999) experiments? We argue that it is not possible to determine whether eye-to-mouth relational properties were used by infants in their study, because the eyeto-lip distance factors were scaled across face stimuli before their average faces were created. Rubenstein et al. do not report whether or not this scaling procedure created a representation of the average female eye-to-mouth distance from a population, nor whether the individual faces were more or less average in comparison with the composite stimuli. Thus, our study shows that infants attend to eye-to-mouth distance properties, whereas Rubenstein et al. cannot make this claim.

A second issue concerns the number of face properties used by infants in the prototype comparison process. Must it be presumed that infant face processing is based on the entire set of "hundreds of dimensions" that were modified to create Rubenstein et al.'s (1999) average face stimuli? Our experiments show that, from the large set of possible face properties, infants are able to discriminate faces based on just a subset of those properties. As other researchers have noted (Farah et al., 1998; Rhodes, Brake, \& Atkinson, 1993), it is difficult to enumerate exactly how many properties define the set, but logic dictates that our digital alteration procedure modified fewer properties than did Rubenstein et al.'s procedure. Specifically, our methodology involved splicing out or adding tiny segments between the eye and the mouth, whereas their 32face averaging methodology involved the entire face. Furthermore, since infants viewed the nonmodified and modified versions of individual face stimuli simultaneously, we controlled looking time variability that did not involve eye-to-mouth relations. However, it must be kept in mind that the set of relational features affected by eye-tomouth distance alterations includes relational properties other than those directly manipulated, such as jawline contour, nose shape, chin-to-eyebrow distance, and lips-tohairline distance. Nevertheless, since Rubenstein et al.'s averaging methodology altered properties that our methodology did not alter (e.g., skin tone, hair color, hair length, hair contour, eye shape, and mouth shape), their face alterations included a larger set of properties and their within-trial comparison stimuli were more different from each other than the stimuli seen by the infants in our experiments.

Further support for our position can be achieved by comparing effect sizes between the present study and published studies employing "averaged" faces and similar experimental paradigms. If our effect sizes are smaller than those reported in Rubenstein et al. (1999), this would be consistent with the argument that a smaller amount of variance is explained by eye-to-mouth distance relations than the variance explained by the averaging methodology they employed.If, on the other hand, our effect sizes are the same as or greater than theirs, this would be consistent with the argument that a particular subset of relations captures most of the variance in infant looking times. Following Cohen (1977), effect sizes were computed for the contrasts reported in the present Experiments 1 and 2, following the procedures outlined to determine effect sizes for nonindependent means. In Experiment 1, the effect size for the difference between nonmodified and modified face pairs was .36. In Experiment 2, the effect size for the difference between unusually long (but nonmodified) and modified faces was .64 , and it was .56 for the difference between nonmodified and shortened face pairs. Using the smallest standard deviations from pairs of means reported in Rubenstein et al. (1999, Experiment 3), the effect sizes are similar, ranging from .37 to $.41 .^{1}$ Thus, we argue that a narrower subset of theoretically possible dimensions are needed to describe face processing in infants than could be concluded from previous research.

Interestingly, when we computed the effect size for the contrast between averaged "seen" and averaged "unseen" faces for the 34-h-old infants in Walton and T. G. R. Bower's (1993) study, the effect size was determined to be 1.21 , which is two to three times the magnitude of the effects just reported. ${ }^{2}$ It is tempting to postulate that, whatever the particular face dimensions encoded, they have far more influence on neonates' face perception than the relations used to compare faces with a prototype in 7-monthold infants.

We cannot determine, on the basis of our research, whether infants can also discriminate faces based on a different subset of properties from within the larger set of theoretically possible face properties. It is a question for future research to decide whether or not particular parameters are given more weighting, or are ignored altogether, in the computational process. One study found that Caucasian infants preferred both the attractive Caucasian and the attractive Black female faces to unattractive faces of either ethnicity (Langlois et al., 1991), suggesting that skin color and perhaps nose shape and hair texture/shape may not be attended to in the prototype comparison process.

It has been known for some time that infants are capable of abstracting prototypical information from schematic faces (Strauss, 1979). The ability that infants show in applying an averaging tool to more realistic face stimuli is per- 
haps not unexpected. Newborn infants are capable of forming a prototype of faces they have seen in less than 1 min (Walton \& T. G. R. Bower, 1993). If they are capable of this from the beginning, what might be said about the development of face processing? We can offer only our speculations.

It is possible that the development of face processing follows a general trend in perceptual development revealed using object discrimination tasks. Thompson and Markson (1998) argue that, between early childhood and adulthood, perception becomes more finely tuned to the slight variations between similar objects. As children get older, they attend to a wider range of dimensionaldifferences between objects, and they become better able to notice slight variations in the colors, shapes, and sizes of objects. Moreover, children become faster at noticing object differences. Applied to the development of face processing, this could mean that children learn to incorporate more second-order relational properties into their face prototypes as they get older, and they could become faster and more adept at noticing slight variations between these second-order properties of different faces.

A second possible avenue of development is that many subprototypes are formed after the original average prototype, which would represent different value judgments of attractiveness commonly made by people. For example, perhaps there is a prototype for an "attractive" young adult female, preferred because it is more commonly experienced. Subsequent to this prototype, another might be created for the less commonly experienced "super-attractive" one. Alley and Cunningham (1991) argue that some extreme facial characteristics are found to be more attractive than average characteristics. In support, research shows that adults prefer female faces that have shortened eye-to-chin distances (Cunningham, Roberts, Barbee, Druen, \& Wu, 1995; Johnston, 2000; Johnston \& Franklin, 1993; Perrett, May, \& Yoshikawa, 1994). Such preferences are possible if, like infants, adults compare second-order relational properties of a perceived face to a prototypical representation.

We further speculate that the eye-to-mouth structural relationships preferred by individuals might be at least partly dependent on timing factors related to hormonal development, particularly during puberty. If, as Johnston (2000) claims, female beauty is a product of sexual selection favoring facial (and body) features that are reliable cues to fertility, one would expect that the male and the female preference for shorter-than-average female jaw lengths might not be apparent until puberty. This possibility is currently being explored.

In the human face, the first-order configural relationship between the eyes and the mouth does not vary (Diamond \& Carey, 1986), yet the distances between these internal features do vary between faces (Farkas, 1981). Thus, an optimal strategy for infants to use in face recognition would be to compare faces in terms of the distances between internal face features. Furthermore, eye-movement research on infants as young as 2 months suggests that infants direct their attention to the eyes and the mouth (Salapatek,
1975). However, one caveat is to be made here: Although eye-movement research shows that young infants' eyes are positioned to encode the eyes and mouth features, their attention is not necessarily exactly at those locations.

Further research is also likely to reveal much about how expertise in face processing is gained during childhood. Although faces may be special to newborn babies in the sense that they pay greater attention to them than to similar objects with scrambled face features (Johnson et al., 1991), they may not be a special class of objects in terms of the nature of developmental growth processes, or even the speed with which full development in those growth processes is reached. Taking a developmental path of subtle, but measurable, changes in perception (Thompson \& Markson, 1998), it nevertheless might take 10 years to encode faces as an expert would (Diamond \& Carey, 1986). There are, as yet, many disagreements among researchers as to how faces are recognized by adults, and whether or not face processing is truly "special” (cf. Farah et al., 1998).

In summary, the present study supports these conclusions: (1) 7-month-old infants attend to particular secondorder relational properties of a face, specifically, those involved in making alterations in the eye-to-mouth distances, and (2) they exhibit a preference for faces closest to an average structural relationship.

\section{REFERENCES}

Alley, T. R., \& Cunningham, M. R. (1991). Averaged faces are attractive, but very attractive faces are not average. Psychological Science, 2, 123-125.

Barrera, M. E., \& Maurer, D. (1981). Recognition of mother's photographed face by the three-month-old. Child Development, 52, 558563.

Bushnell, I. W. R., SAI, F., \& Mullin, J. T. (1989). Neonatal recognition of the mother's face. British Journal of Developmental Psychology, 7, 3-15.

Carey, S., \& Diamond, R. (1977). From piecemeal to configurational representation of faces. Science, 195, 312-314.

Carey, S., \& Diamond, R. (1999). Are faces perceived as configurations more by adults than by children? Visual Cognition, 1, 253-274.

Carey, S., Diamond, R. \& Woods, B. (1980). Development of face recognition-a maturational component? Developmental Psychology, 16, 257-269.

COHEN, J. (1977). Statistical power analysis for the behavioral sciences. New York: Academic Press.

Cunningham, M. R, Roberts, A. R., Barbee, A. P., Druen, P. B., \& Wu, C. (1995). Their ideas of beauty are, on the whole, the same as ours: Consistency and variability in the cross-cultural perception of female physical attractiveness. Journal of Personality \& Social Psychology, 68, 261-279.

de HaAn, M., \& Nelson, C. A. (1997). Recognition of the mother's face by six-month-old infants: A neurobehavioral study. Child Development, 68, 187-210.

DiAmond, R., \& CAREY, S. (1986). Why faces are and are not special: An effect of expertise. Journal of Experimental Psychology: General, 115, 107-117.

Ericsson, K. A., \& SMith, J. (1991). Prospects and limits of the empirical study of expertise: An introduction. In K. A. Ericsson \& J. Smith (Eds.), Toward a general theory of expertise: Prospects and limits. Cambridge: Cambridge University Press.

Farah, M. J., Wilson, K. D., Drain, M., \& Tanaka, J. N. (1998). What is "special" about face perception? Psychological Review, 105, 482-498.

FARKAS, L. G. (1981). Anthropometric facial proportions in medicine. Springfield, IL: C. C. Thomas. 
Field, T. M., Cohen, D., Garcia, R., \& Greenberg, R. (1984). Mother-stranger face discrimination by the newborn. Infant Behavior \& Development, 7, 19-25.

JAMES, W. (1890). Principles of psychology (Vol. 1). New York: Holt. Johnson, M. H., Dziurawiec, S., Ellis, H., \& Morton, J. (1991). Newborns' preferential tracking of face-like stimuli and its subsequent decline. Cognition, 40, 1-19.

Johnston, V. S. (2000). Female facial beauty: The fertility hypothesis. Pragmatics \& Cognition, 8, 107-122.

Johnston, V. S., \& Franklin, M. (1993). Is beauty in the eye of the beholder? Ethology \& Sociobiology, 14, 183-199.

LANGlois, J. H., Ritter, J. M., Roggman, L. A., \& VAughn, L. S. (1991). Facial diversity and infant preferences for attractive faces. Developmental Psychology, 27, 79-84.

Langlois, J. H., \& Roggman, L. A. (1990). Attractive faces are only average. Psychological Science, 1, 115-121.

Langlois, J. H., Roggman, L. A., Casey, R. J., Ritter, J. M., RieserDANner, L. A., \& Jenkins, V. Y. (1987). Infant preferences for attractive faces: Rudiments of a stereotype? Developmental Psychology, 23, 363-369.

Langlois, J. H., Roggman, L. A., \& Musselman, L. (1994). What is average and what is not average about attractive faces? Psychological Science, 5, 214-220.

Pascalis, O., De Schonen, S., Morton, J., Deruelle, C., \& FabreGrenet, M. (1995). Mother's face recognition by neonates: A replication and an extension. Infant Behavior \& Development, 18, 79-85.

Perrett, D. I., May, K. A., \& Yoshikawa, S. (1994). Facial shape and judgments of female attractiveness. Nature, 368, 239-242.

RHodEs, G. (1988). Looking at faces: First-order and second-order features as determinates of facial appearance. Perception, 17, 43-63.

Rhodes, G., Brake, S., \& Atkinson, A. P. (1993). What's lost in inverted faces? Cognition, 47, 25-57.

Rhodes, G., BRENNAN, S., \& CAREY, S. (1987). Identification and ratings of caricatures: Implications for mental representations of faces. Cognitive Psychology, 19, 473-497.

Rhodes, G., Sumich, A., \& Byatt, G. (1999). Are average facial configurations attractive only because of their symmetry? Psychological Science, 10, 52-58.

Rhodes, G., \& Tremewan, T. (1996). Averageness, exaggeration, and facial attractiveness. Psychological Science, 7, 105-110.

Rubenstein, A. J., KalaKanis, L., \& Langlois, J. H. (1999). Infant preferences for attractive faces: A cognitive explanation. Developmental Psychology, 35, 848-855.

Salapatek, P. (1975). Pattern perception in early infancy. In L. B. Cohen
\& P. Salapatek (Eds.), Infantperception: From sensation to cognition. New York: Academic Press.

Samuels, C. A., Butterworth, G., Roberts, T., Graupner, L., \& Hole, G. (1994). Babies prefer attractiveness to symmetry. Perception, 23, 823-831.

Samuels, C. A., \& Ewy, R. (1985). Aesthetic perception of faces during infancy. British Journal of Developmental Psychology, 3, 221228.

Slater, A., Von der Schulenburg, C., Brown, E, Badenoch, M., Butterworth, G., Parson, S., \& Samuels, C. (1998). Newborn infants prefer attractive faces. Infant Behavior \& Development, 21, 345354.

STRAUSS, M. S. (1979). Abstraction of prototypical information by adults and 10-month-oldinfants. Journal of Experimental Psychology: Human Learning \& Memory, 5, 618-632.

Thompson, L. A., \& MArkson, L. (1998). Developmental changes in the effect of dimensional salience on the discriminability of object relations. Journal of Experimental Child Psychology, 69, 1-25.

Valentine, T., \& Bruce, V. (1986). The effects of distinctiveness in recognising and classifying faces. Perception, 15, 525-535.

WAlton, G. E., \& Bower, T. G. R. (1993). Newborns form "prototypes" in less than 1 minute. Psychological Science, 4, 203-205.

Walton, G. E., Bower, N. J. A., \& Bower, T. G. R. (1992). Recognition of familiar faces by newborns. Infant Behavior \& Development, 15 , 265-269.

YIN, R. K. (1969). Looking at upside-down faces. Journal of Experimental Psychology, 81, 141-145.

\section{NOTES}

1. In this experiment, infants were shown to discriminate novel from averaged faces, and familiar from averaged faces, but they actually did not prefer averaged faces. In Experiment 2, infants' looking time preferences favored the averaged over unattractive faces; however, standard deviations were not reported. These are necessary in computing effect sizes for the difference between means.

2. Since correlations between mean difference scores were not reported for any of the contrasts, we followed Cohen's (1977) recommended procedure of computing effect sizes for independent means. The test is robust for cases of equal variances across conditions.

(Manuscript received February 1, 2000; revision accepted for publication March 26, 2001.) 